\title{
A melanocortin 1 receptor $(M C 1 R)$ gene polymorphism is useful for authentication of Massese sheep dairy products
}

\author{
Luca Fontanesi ${ }^{1 *}+$, Francesca Beretti ${ }^{1,2}+{ }^{2}$, Stefania Dall'Olio ${ }^{1}$, Baldassare Portolano ${ }^{2}$, \\ Donato Matassino ${ }^{3}$ and Vincenzo Russo ${ }^{1}$ \\ ${ }^{1}$ DIPROVAL, Sezione di Allevamenti Zootecnici, University of Bologna, Via F.lli Rosselli 107, 42123 Reggio Emilia, Italy \\ ${ }^{2}$ Dipartimento S.En.Fi.Mi.Zo., Sezione di Produzioni Animali, University of Palermo, Viale delle Scienze - Parco d'Orleans, 90128 Palermo, \\ Italy \\ ${ }^{3}$ ConSDABI, Contrada Piano Cappelle, 82100 Benevento, Italy
}

Received 12 May 2010; accepted for publication 26 October 2010

\begin{abstract}
Massese is an Italian sheep breed, with black or grey coat colour, mainly reared in the Tuscany and Emilia Romagna regions. Recently, the emerging interests in this breed have resulted in the production of Pecorino cheese obtained with only Massese milk. In order to be profitable, this marketing link between Massese breed and its products should be defended against fraudsters who could include milk of other sheep breeds or cow milk in Massese labelled productions. To identify the genetic factors affecting coat colour in sheep, we have recently analysed the melanocortin 1 receptor $(M C 1 R)$ gene and identified several single nucleotide polymorphisms (SNPs). In this work, as a first step to set up a DNA based protocol for authentication of Massese dairy products, we further investigated the presence and distribution of one of these SNPs (c.-31G >A) in 143 Massese sheep and in another 13 sheep breeds (for a total of 351 animals). The Massese breed was fixed for allele c.-31A, whereas in all other breeds allele c.-31 G was the most frequent or with frequency of $0 \cdot 50$. At the same nucleotide position the cattle $M C 1 R$ gene carries the $G$ nucleotide. Using these data we developed a method to detect adulterating milk (from other sheep breeds or from cow) in Massese dairy products based on the analysis of the c.-31G > A SNP. We first tested the sensitivity of the protocol and then applied it to analyse DNA extracted from ricotta and Pecorino cheese obtained with only Massese milk or obtained with unrestricted sheep and cattle milk. To our knowledge, this system represents the first one that can be used for breed authentication of a sheep production and that, at the same time, can reveal frauds derived from the admixture of milk of an unreported species.
\end{abstract}

Keywords: authentication, Massese, MC1R, mono-breed products, sheep breeds, SNP.

Massese is an Italian sheep breed, with black or grey coat colour, mainly reared in the Tuscany and Emilia Romagna regions. It accounts for about 5,000 animals registered to the herdbook. Massese is considered a dairy breed even if its importance for lamb meat has increased during the last years. Recently, emerging interests in this breed have resulted in the production of Pecorino cheese obtained with only Massese milk and in marketing heavy lamb meat of Massese breed. These mono-breed labelled products have been developed in order to differentiate productions and, in turn, to improve profitability with expected impacts on rural economy of marginal areas and conservation of ovine

*For correspondence; e-mail: luca.fontanesi@unibo.it tAuthors Fontanesi \& Beretti ${ }^{1}$ contributed equally to this work biodiversity, as already achieved by several other dairy and meat mono-breed animal products (Fontanesi 2009). However, in order to be profitable, this marketing link between Massese breed and its products should be defended against fraudsters who include milk or lamb meat of other sheep breeds in Massese labelled productions.

Another important problem of ovine dairy products is fraud involving the use of unreported cow milk mixed with ovine milk. This fraud can be easily detected with the use of conventional or DNA based methods, therefore the application of protocols to evidence the presence of cow milk in ovine dairy products (species identification) is just a matter of sensitivity and practical implementation of specific protocols (e.g. Moio et al. 1990; Anguita et al. 1997; Bottero et al. 2003). On the contrary, the identification of the breed of origin of animal products still remains a challenge in all farm 
animal species, including sheep. In general, if the question is at the breed level it is better to use the terms of breed authentication because what is requested is to confirm that the claims about the breed of origin of the products are true. Two approaches that use DNA marker information have been proposed for breed authentication: i) a probabilistic approach, mainly based on the use of highly variable microsatellites, AFLP markers or tens of single nucleotide polymorphisms (SNPs) combined with different computational analyses that assign individuals to a particular breed with a certain probability (e.g. Ciampolini et al. 2000; Maudet et al. 2002; Negrini et al. 2009); ii) a deterministic approach, based on the use of a few breed specific or exclusive markers, whose usefulness is due only to their fixation or absence in the considered breeds and, as a consequence, in the analysed products (e.g. Maudet \& Taberlet 2002; D'Alessandro et al. 2007; Russo et al. 2007). It is quite clear that the probabilistic approach cannot be applied for breed authentication of dairy products, because they usually derive from milk of more than one animal. On the contrary, the deterministic approach can be applied to a mixture of products obtained from more animals as it relies on the identification of markers that are present or absent in all (or most) animals of a particular breed. However, the main problem for this approach is the identification of DNA markers with these characteristics. Useful markers for this purpose can be identified by looking at mutations in genes determining the most important traits that differentiate the breeds, like coat colour (Russo \& Fontanesi 2004; Fontanesi 2009).

A large number of genes affect coat colour in mammals but two main loci (Agouti and Extension) are the major determinants of the relative amounts of black/brown (eumelanin) and red/yellow (phaeomelanin) pigments produced in melanocytes (Searle 1968). These loci encode for the agouti signalling protein $(A S I P)$ and melanocortin 1 receptor $(M C 1 R)$ genes, respectively (Bultman et al. 1992; Robbins et al. 1993). Mutations in these genes have been shown to affect coat colours in a large number of mammals, including several farm animal species like cattle (Klungland et al. 1995; Girardot et al. 2006), pig (Kijas et al. 1998), horse (Marklund et al. 1996), goat (Fontanesi et al. 2009a, b), and rabbit (Fontanesi et al. 2006, 2010b). Mutations in the ASIP and MC $1 R$ genes have been also shown to affect coat colour in sheep (Våge et al. 1999; Norris \& Whan, 2008; Royo et al. 2008; Fontanesi et al. 2011). We recently re-sequenced the $M C 1 R$ gene in several sheep breeds and confirmed the presence of two missense mutations (p.M73 K and p.D121 N) causing the dominant black Extension $\left(E^{D}\right)$ allele. We also identified a novel MC1R missense mutation (p.R67C) that we suggested to cause the recessive e allele at the ovine Extension series (Fontanesi et al. 2010a). In addition, we determined four MC1R haplotypes derived from the combination of seven SNPs. Haplotype 3 was the most frequent in the Massese sheep breed that also presented haplotype 4, the latter included the two mutations causing the $E^{D}$ allele. Both haplotypes can be distinguished from the others by the common presence of a polymorphism (c.-31G > A) in the 5'-untranslated region (5'-UTR) (Fontanesi et al. 2010a).

As a first step to identify a DNA marker that could be useful for authentication of Massese sheep dairy products, we further investigated the presence and distribution of the MC1R c.-31G > A SNP in a larger number of Massese sheep and in several other sheep breeds. Moreover, analysing this SNP, we set up a protocol to investigate admixture of milk of different sheep breeds and/or cattle milk in Massese dairy products. To our knowledge, this system represents the first one that can be used for breed and species authentication at the same time.

\section{Materials and Methods}

\section{Animals and dairy products}

Blood, milk and/or hair were collected from 351 sheep of 14 different breeds (Table 1). Massese, Sarda, and Valle del Belice (the breeds with the larger number of samples) sheep were sampled from four, three, and three different flocks in the North of Italy (Massese and Sarda) and in Sicily (Valle del Belice), respectively. Samples from all other breeds were collected from one or two flocks or were obtained from animals analysed for pedigree verification by the Italian Breeder Association laboratory. In addition, hair or milk samples were collected from five Italian Holstein and five Italian Brown cattle.

Three samples of Pecorino cheese (3-6 months old) and one sample of ricotta cheese obtained with only Massese milk were purchased directly from the producers. Another cheese sample, purchased directly from the producer (3 months old), was prepared with Massese milk mixed with about $20 \%$ cows' milk. Three other Pecorino cheeses (4-6 months old) not labelled as mono-breed products were purchased in retailer markets of North Italy together with a sample of cheese produced with both ovine and bovine milk (3 months old). These products were considered as obtained from undefined sheep without breed limitation.

\section{DNA extraction}

DNA from individual samples was extracted using a standard phenol-chloroform protocol for blood, the Wizard ${ }^{\circledR}$ Genomic DNA Purification kit for blood and milk (Promega Corporation, Madison, WI, USA) or rapid extraction methods for milk and hair roots (Russo et al. 2007). For dairy products (cheese and ricotta cheese), DNA was extracted using a commercial kit designed for blood (QIAamp DNA Blood Midi Kit, Qiagen) as reported by Russo et al. (2007). Briefly, about 1-2 g cheese was homogenized in a $15 \mathrm{ml}$ tube. Then, $4 \mathrm{ml}$ bi-distilled water and $200 \mu \mathrm{l}$ of a $20 \mathrm{mg}$ proteinase $\mathrm{K} / \mathrm{ml}$ solution were added. This mixture was vortexed and placed overnight in a rocking oven at $60^{\circ} \mathrm{C}$. Subsequently, about $2 \mathrm{ml}$ was used for DNA extraction according to the manufacturer's protocol. 
Table 1. Allele and genotype frequencies observed in different sheep breeds for the MC1R c.-31G >A SNP.

\begin{tabular}{|c|c|c|c|c|c|c|}
\hline \multirow[b]{2}{*}{ Breed } & \multirow[b]{2}{*}{ No. of sheept } & \multicolumn{3}{|c|}{ Genotypes } & \multicolumn{2}{|c|}{ Allele frequencies } \\
\hline & & c. $-31 \mathrm{GG}$ & c. $-31 \mathrm{GA}$ & c. $-31 \mathrm{AA}$ & c. $-31 \mathrm{G}$ & C. $-31 \mathrm{~A}$ \\
\hline Appenninica & 14 & 4 & 7 & 3 & $0 \cdot 54$ & $0 \cdot 46$ \\
\hline Bagnolese & 13 & 8 & 5 & - & $0 \cdot 81$ & $0 \cdot 19$ \\
\hline Barbaresca & 16 & 14 & 2 & - & 0.94 & $0 \cdot 06$ \\
\hline Bergamasca & 8 & 4 & 4 & - & 0.66 & $0 \cdot 34$ \\
\hline Comisana & 21 & 8 & 11 & 2 & $0 \cdot 64$ & $0 \cdot 36$ \\
\hline Cornigliese-like & 10 & 8 & 1 & 1 & $0 \cdot 80$ & $0 \cdot 20$ \\
\hline Delle Langhe & 6 & 1 & 5 & - & $0 \cdot 75$ & $0 \cdot 25$ \\
\hline Massese & 143 & - & - & 143 & 0.00 & $1 \cdot 00$ \\
\hline Laticauda & 10 & 4 & 3 & 3 & $0 \cdot 55$ & $0 \cdot 45$ \\
\hline Merinizzata Italiana & 4 & 2 & 1 & 1 & 0.63 & $0 \cdot 37$ \\
\hline Moscia Leccese & 5 & 2 & 3 & - & $0 \cdot 70$ & $0 \cdot 30$ \\
\hline Pinzirita & 6 & 1 & 4 & 1 & $0 \cdot 50$ & $0 \cdot 50$ \\
\hline Sarda & 43 & 25 & 16 & 2 & $0 \cdot 74$ & $0 \cdot 26$ \\
\hline Valle del Belice & 52 & 34 & 13 & 5 & $0 \cdot 78$ & $0 \cdot 22$ \\
\hline Total & 351 & & & & & \\
\hline
\end{tabular}

+All breeds with more than 20 genotyped animals were in Hardy-Weinberg equilibrium $(P>0.05)$

\section{Analysis of a MC1R polymorphism in individual samples and in dairy products by PCR-RFLP}

A polymorphism (c.-31G $>$ A) located in the $5^{\prime}-U T R$ of the sheep MC1R gene (Fontanesi et al. 2010a) was genotyped by PCR-RFLP. PCR was performed using a TGradient thermal cycler (Biometra, Göttingen, Germany) or a PTC-100 thermal cycler (MJ Research, Watertown, MA, USA) in a volume of $20 \mu \mathrm{l}$ containing 10-50 ng DNA template, $1 \mathrm{U}$ DNA EuroTaq DNA polymerase (EuroClone Ltd., Paington, Devon, UK), 1X PCR Buffer, 2.5 mm-dNTPs, 10 pmol of each primer (primer forward: 5'-gcaactgcacatccagagaa-3'; primer reverse: 5'-cgcagggagcaggaaagggtgctag- $\left.3^{\prime}\right)$, and $2.0 \mathrm{~mm}-$ $\mathrm{MgCl}_{2}$. PCR profile was as follows: $5 \mathrm{~min}$ at $95{ }^{\circ} \mathrm{C} ; 35$ amplification cycles of $30 \mathrm{~s}$ at $95{ }^{\circ} \mathrm{C}, 30 \mathrm{~s}$ at $58{ }^{\circ} \mathrm{C}, 30 \mathrm{~s}$ at $72{ }^{\circ} \mathrm{C} ; 5 \mathrm{~min}$ at $72{ }^{\circ} \mathrm{C}$. The reverse primer contained a mismatched nucleotide (underlined base) that inserted an additional artificial restriction site for endonuclease Alul when allele c.-31 G was amplified. The obtained PCR product of $202 \mathrm{bp}$ was digested with $3 \mathrm{U}$ of Alul (Fermentas, Vilnius, Lithuania) and the resulting DNA fragments were electrophoresed in 10\% polyacryamide:bisacrylamide 29:1 TBE 1X gels. DNA fragments were visualized with $1 \mathrm{X}$ GelRed Nucleid Acid Gel Stain (Biotium Inc., Hayward, CA, USA). Electrophoretic patterns of the different genotypes are presented in Fig. 1. Evaluation of Hardy-Weinberg equilibrium of the analysed polymorphism was obtained with chi-square analysis from the HWE software program (Linkage Utility Programs, Rockefeller University, New York, NY, USA) only for breeds with more than 20 genotyped animals.

\section{Analysis of the PCR-RFLP protocol on cattle genomic DNA}

Cattle and sheep MC1R gene sequences (GenBank accession numbers: AF445642 and FN600555, respectively) were
M GA AA AA GA GG GG GG GG GG AA

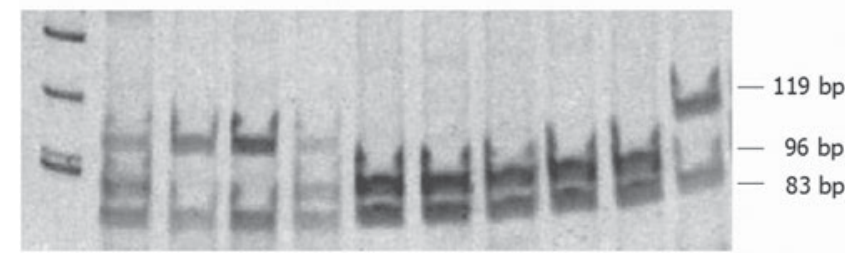

Fig. 1. PCR-RFLP analysis with Alul of the MC1R c.-31G $>$ A SNP in different sheep. The genotype of each animal is reported at the top of the corresponding gel line. $G G=$ c.-31GG; $G A=$ c.-31GA; $\mathrm{AA}=\mathrm{c} .-31 \mathrm{AA} ; \quad \mathrm{M}=\mathrm{DNA}$ molecular ladder (ZR $50 \mathrm{bp}$ DNA Marker $^{\mathrm{TM}}$; Zymo Research, Orange, CA, USA). The fragment of 23 bp produced by the digestion of the allele c.-31 G fragment is not shown in the gel.

aligned with bl2seq BLAST program http://blast.ncbi.nlm. nih.gov/Blast.cgi showing 95\% identity. At the same position of the polymorphic site in sheep, the cattle $M C 1 R$ sequence reports $G(c .-31 G)$, the nucleotide that was not identified in the Massese breed. Therefore the PCR-RFLP protocol described above was used to analyse this nucleotide position on cattle genomic DNA isolated from individual cattle samples or from two samples of cheese produced with both ovine and bovine milk.

\section{Evaluation of the sensitivity of the PCR-RFLP protocol}

In order to have a first rough estimation of the sensitivity of the PCR-RFLP allele detection method in sheep dairy products, we adapted and combined the methods described by Breem et al. (2000) and Maudet \& Taberlet (2001) and already applied by Russo et al. (2007) for the authentication of Reggiana cattle Parmigiano Reggiano cheese. Several mixtures of DNA including different proportions of the two alleles of the c.-31G >A SNP (0:100, 5:95, 10:90, 25:75, 
50:50, 75:25, and 100:0) were prepared combining genomic DNA of homozygous c.-31GG and c.-31AA sheep. For each mixture, two aliquots were prepared independently. DNA concentration was evaluated using a NanoDrop ND-1000 spectrophotometer (NanoDrop Technologies Inc., Wilmington, DE, USA). Restriction analysed fragments were electrophoresed and stained as reported above. Bands were captured using a Kodak EDAS 290 instrument (Eastman Kodak Company, Scientific Imaging Systems, Rochester, NY, USA). Band peak intensity and band net intensity where obtained using the Kodak 1D image analysis software (Eastman Kodak Company). The relative intensity (RI) was calculated as the averaged ratio of the band peak intensity and band net intensity obtained for the $119 \mathrm{bp}$ and $96 \mathrm{bp}$ fragments.

\section{Results and Discussion}

\section{Analysis of the MC1R c.-31G > A SNP in different sheep breeds}

A previous study analysed $M C 1 R$ as a candidate gene for coat colour in Massese and several other sheep breeds (Fontanesi et al. 2010a). Despite the black coat colour of most Massese sheep, the dominant black $E^{D}$ allele was identified at a very low frequency $(2 \%)$ in this breed (Fontanesi et al. 2010a). Interestingly, the MC1R $E^{D}$ haplotype (haplotype 4) derived from haplotype 3, which shared 5 out of 7 polymorphic sites (only the two missense mutations of the $E^{D}$ allele were not in common). Haplotype 3 was the most frequent in Massese sheep and was considered as a wild-type haplotype (Fontanesi et al. 2010a, 2011). A polymorphic site in the $5^{\prime}-$ UTR of the $M C 1 R$ gene (c.-31G $>A$ ) contributes to differentiate haplotypes 3 and 4 (the only ones that were preliminarily observed in the Massese breed) from haplotype 1 (the most common in other breeds) and haplotype 2 (observed only in Valle del Belice sheep) (Fontanesi et al. 2010a). As these results were obtained for a small number of Massese sheep, we further genotyped the c.-31G > A SNP in a larger number of animals of this breed and confirmed that Massese animals carried only allele c.-31A (at least in the analysed population; Table 1). This result may indicate a possible selection pressure on MC1R haplotypes in Massese sheep probably derived by the characteristic black or grey coat colours. As a matter of fact haplotype 4 causes dominant black coat colour and haplotype 3, being a putative wild-type haplotype, makes the expression of Agouti mutated alleles possible, that are the most important coat colour determinants in this breed (Fontanesi et al. 2011).

The c.-31A allele was also identified in all other investigated Italian sheep breeds, but with lower frequencies, ranging from 0.06 to 0.50 (Table 1 ). In particular, in the Sarda breed, which is the most common and diffused sheep breed in Italy and, for this reason, could be the source of possible frauds in Massese dairy products, the frequency of allele
C.-31 A was only $0 \cdot 26$. In addition only 2 out of 43 genotyped animals $(0.046)$ of this breed had genotype c.-31AA. The three $M C 1 R$ genotypes obtained in the Sarda breed were in Hardy-Weinberg equilibrium $(P=0.778)$. In other sheep breeds that share, to some extent, the same (or close) areas of diffusion of Massese sheep (Appenninica, Bergamasca, Comisana and Cornigliese-like), genotype c.-31AA was not observed or, in general, had a lower frequency (it should be considered that for these breeds only few animals were genotyped) (Table 1). Concerning all other breeds (Bagnolese, Barbaresca, Delle Langhe, Laticauda, Merinizzata Italiana, Moscia Leccese, Pinzirita and Valle del Belice), it should be highly improbable that Massese products are obtained with admixture of milk of these other breeds for the facts that: i) some of them are considered local breeds and, for this reason, they are not very common: on the other hand, they might be even interested in defending/establishing their own mono-breed products; ii) some breeds, in addition, are not reared in the regions of Massese sheep and, for this reason, their milk might not be used for frauds; iii) some breeds are considered meat (Bergamasca) or dual purpose breeds (meat/wool: Merinizzata Italiana; meat/milk: Bagnolese, Barbaresca and Laticauda) and are not usually used to produce dairy products, or their dairy products are commercialised only in local markets.

\section{Application of the MC1R c.-31G > A polymorphism for authentication of Massese dairy products}

Considering the genotyping results of the $M C 1 R$ c.-31G $>A$ SNP in Massese and all other investigated sheep breeds, only for the fact that Massese is fixed for one allele, it is possible to consider this marker for authentication purposes. However, as the analysed animals represent only a fraction of the Massese population, we cannot exclude the possibility of the existence of the other allele in the Massese breed. Nevertheless, its frequency would be very low and would not preclude the use of this marker to exclude, at least in part, the presence of milk of other breeds in Massese dairy products as reported for similar applications (Fontanesi 2009). As discussed above, frauds could be obtained mainly from admixture with Sarda milk. It is highly improbable that all sheep of a flock of this breed can have the same genotype of the Massese sheep. In the PCR-RFLP analysis, the detection of the DNA fragment corresponding to allele c.-31 G may indicate admixture with milk of Sarda (or other breeds). In addition, as the cattle MC1R gene can be amplified with the same primers and the PCR-RFLP analysis of the obtained fragment produces the same pattern of the sheep c.-31 G allele, the same protocol can detect admixture of cattle milk in Massese products. Therefore, the protocol can test if there is milk that does not come from Massese sheep. However, it is not possible to distinguish if the fraud derives from milk of other sheep breeds or from cow milk. In these cases, additional analyses are needed to establish if the fraud is at the species level (e.g. Moio et al. 1990; Anguita et al. 1997; 
Bottero et al. 2003). Similar reasoning can be reported for breed authentication of lamb meat. In this case, it should be considered that the tested product is derived from one animal and not from more animals that in general are the original source of dairy products. For lamb meat authentication, it could be possible to apply a probabilistic approach based on microsatellites. Using microsatellites Bozzi et al. (2009) provided reliable allocation of Massese sheep against several other local breeds. Their results could be useful to establish a more comprehensive method for sheep breed allocation that can be used for breed authentication of lamb meat. This probabilistic approach could benefit from the analysis of the MC1R c.-31G $>$ A SNP, given its high discriminatory power for Massese products.

An important step to evaluate the practical usefulness of the PCR-RFLP method to identify the presence of the c.-31 G allele or base position (considering the cattle MC1R gene) was the analysis of the sensitivity of this test. The evaluation of the sensitivity of the genotyping protocol was intended as the identification of the lower detection limit of possible contaminating DNA, namely alien DNA (DNA of not allowed breeds or species), in the analysed products. This issue was investigated using as PCR template mixtures of DNA including different proportions of DNA of animals with diverse $M C 1 R$ c.-31G $>$ A genotype. The use of already extracted DNA instead of mixture of milk or purposely manufactured cheese containing known proportions of milk of animals with different MC1R genotype overcomes the problem due to the large variation in somatic cell count of different milk samples. Figure 2 reports the plotted RI of the $119 \mathrm{bp}$ and $96 \mathrm{bp}$ DNA fragments (c.-31A and c.-31 G alleles, respectively) for the analysed artificial constructed reference samples. The c.-30 G allele was evidenced when it was $5 \%$ (capturing the images with the dedicated software), but was clearly visible when it was $10 \%$, indicating that the PCR-RFLP protocol was sensitive enough to detect admixture of disallowed DNA. However, it was not too sensitive to detect possible accidental admixtures (Fig. 2). This sensitivity of $5-10 \%$ could account for the possible very low frequency of allele c.-31 G in a Massese flock, that our allele frequency analysis in the breed could not exclude at priori. The obtained data points could represent a rough semi-quantitative estimation of the level of mixture of DNA with different genotypes. However, to use these reference samples to estimate the level of milk admixture, it should be considered that i) the DNA content of milk (as well as cheese) derives from the somatic cell count of individual milk samples, which is affected by several factors and alters the contribution of the individual DNA to the milk pool from which the dairy products are obtained; and ii) the sensitivity of the mutation detection method could give distorted estimations for alleles at lower concentration (Breem et al. 2000).

The PCR-RFLP protocol was used to analyse DNA extracted from the 8 samples of cheese (3 of only Massese milk, 1 of Massese milk with cow milk, 3 of unknown ovine breeds and 1 of unknown sheep mixed with cow milk) and a)

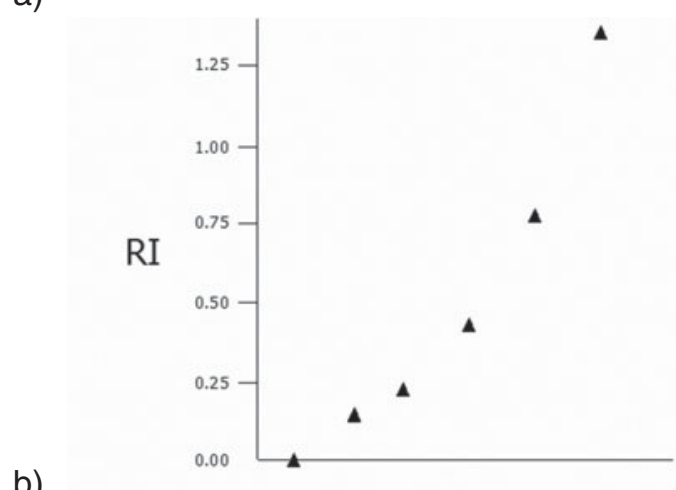

b)

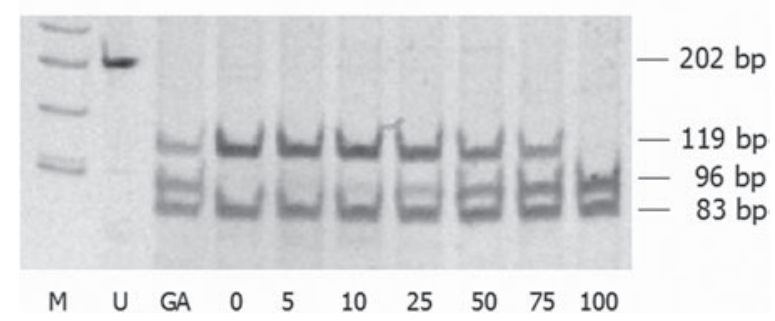

Fig. 2. a) Averaged RI data points (obtained from the two independent analyses) resulted for the PCR-RFLP analysis (Alul) of artificial constructed reference DNA samples containing different concentrations of genomic DNA of a sheep with c.-31GG genotype. The data points are in the correspondence of the reference samples loaded in the gel below it. b) PCR-RFLP gel analysis of artificial constructed reference DNA samples. $\mathrm{RI}=$ relative intensity. M=DNA molecular ladder (ZR 50 bp DNA Marker ${ }^{\mathrm{TM}}$; Zymo Research). $U=$ undigested $P C R$ fragment. $G A=$ sheep with genotype C.-31GA. 0 to $100=$ artificial reference samples with different concentration (from 0 to $100 \%$ ) of genomic DNA of a sheep with genotype c.-31GG.

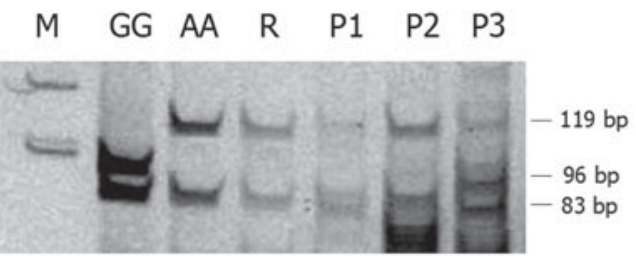

Fig. 3. PCR-RFLP analysis (Alul) of different cheese samples. $\mathrm{M}=$ DNA molecular ladder (ZR $50 \mathrm{bp}$ DNA Marker ${ }^{\mathrm{TM}}$; Zymo Research). GG=sheep with genotype c.-31GG. AA= sheep with genotype c.-31AA. $\mathrm{R}=$ ricotta cheese of only Massese milk. P1 and P2 = Pecorino cheese of only Massese milk. P3 = Pecorino cheese of unknown sheep milk.

from the Massese ricotta cheese. DNA was amplified from 8 out of 9 of these dairy samples. No amplification was obtained from one Pecorino cheese of unknown sheep, even if several attempts (of DNA extraction and PCR) were carried out. The failure to analyse this sample might be due to the low level of somatic cells in the milk used to produce it or to other unknown factors. As expected, all 3 Pecorino and ricotta cheese samples of only Massese milk showed only the presence of the MC1R C.-31A allele (Fig. 3). Allele 
c.-31 G was evidenced in all other cheese samples of unknown sheep and in those including cow milk (Fig. 3 and data not shown). To our knowledge, this protocol is the first one that has been developed for authentication of sheep mono-breed products. In addition, this system can be used, at the same time, to test the admixture of milk of another species.

\section{Problems and limits on using coat colour gene markers for authentication of sheep products}

According to the studies we carried out for the Massese sheep breed, it is possible to derive a few general lessons in the application of coat colour gene markers for breed authentication. First of all, sheep breeds/populations are in several cases not fixed for any coat colour making difficult the identification of DNA markers in coat colour genes having useful characteristics for this purpose (Fontanesi et al. 2010a, 2011). Other phenotypic traits, like muscle mass, could be also considered for the identification of associated markers, but at present only few examples that are not based on coat colour gene polymorphisms are available in other livestock species (Fontanesi 2009). However, with the use of dense SNP panels it could be possible to identify other useful DNA markers in unexpected genomic regions (Fontanesi 2009).

For breed authentication, the optimal situation would be if an allele is present (or absent) only in the breed of interest. This case seems quite rare even for mutations determining coat colour as also observed in other livestock species (D'Alessandro et al. 2007; Russo et al. 2007; Fontanesi 2009). Mutations that produce recessive coat colour or for which their phenotypic effect is covered by epistatic mechanisms can remain in the population even if their effect is not evident. In addition, the processes that contributed to the constitution of the breeds (bottlenecks, reproductive isolation, crossbreeding, introgression, etc.) shaped allele frequency distribution of neutral or partially functional polymorphisms (or markers in linkage disequilibrium with causative mutations) but maintained in most cases (at different frequencies) the same alleles in different populations. This seems the case we reported for the MC1R c.-31G $>A$ SNP that despite the fixation of the c.-31A allele in the Massese sheep (according to the analysed samples), this allele is also present in other breeds. Therefore the use of this polymorphic site in Massese product authentication (as well as in similar other cases) should be evaluated according to this fact. Moreover, geographical distribution of other breeds compared with the target breed (Massese in our study) should be considered when the results of implemented tests are interpreted. It is almost impossible that milk (or meat) of other local breeds (with small population size) or breeds traditionally reared in distant regions from the target breed are used to counterfeit or produce frauds. However, in general, cautions should be always used before accepting the result of tests based on DNA markers with these characteristics as a final proof.
We thank Dr. Michele Blasi (AIA, Roma, Italy) and several farmers for providing samples. This work was supported by MiPAAF Selmol project.

\section{References}

Anguita G, Martín R, García T, Morales P, Haza Al, González I, Sanz B \& Hernández PE 1997 A competitive enzyme-linked immunosorbent assay for detection of bovine milk in ovine and caprine milk and cheese using a monoclonal antibody against bovine $\beta$-casein. Journal of Food Protection 60 64-66

Bottero MT, Civera T, Nucera D, Rosati S, Sacchi P \& Turi RM 2003 A multiplex polymerase chain reaction for the identification of cows', goats' and sheep's milk in dairy products. International Dairy Journal 13 277-282

Bozzi R, Degl'Innocenti P, Rivera Diaz P, Nardi L, Crovetti A, Sargentini C \& Giorgetti A 2009 Genetic characterization and breed assignment in five Italian sheep breeds using microsatellite markers. Small Ruminant Research 85 50-57

Breem G, Harold D, Ralston S, Shaw D \& St. Clair D 2000 Determining SNP allele frequencies in DNA pools. Biotechniques $28464-470$

Bultman SJ, Michaud EJ \& Woychik RP 1992 Molecular characterization of the mouse agouti locus. Cell 71 1195-1204

Ciampolini R, Leveziel H, Mozzanti E, Grohs C \& Cianci D 2000 Genomic identification of the breed of an individual or its tissue. Meat Science $\mathbf{5 4}$ 34-40

D'Alessandro E, Fontanesi L, Liotta L, Davoli R, Chiofalo V \& Russo V 2007 Analysis of the MC1R gene in the Nero Siciliano pig breed and usefulness of this locus for breed traceability. Veterinary Research Communication 31 (Suppl. 1) 389-392

Fontanesi L 2009 Genetic authentication and traceability of food products of animal origin: new developments and perspectives. Italian Journal of Animal Science 8 (Suppl. 2) 9-18

Fontanesi L, Beretti F, Riggio V, Dall'Olio S, Gómez González E, Finocchiaro R, Davoli R, Russo V \& Portolano B 2009a Missense and nonsense mutations in melanocortin 1 receptor $(M C 1 R)$ gene of different goat breeds: association with red and black coat colour phenotypes but with unexpected evidences. BMC Genetics 1047

Fontanesi L, Beretti F, Riggio V, Gómez González E, Dall'Olio S, Davoli R, Russo V \& Portolano B 2009b Copy number variation and missense mutations of the agouti signaling protein $(A S I P)$ gene in goat breeds with different coat colours. Cytogenetic and Genome Research 126 333-347

Fontanesi L, Beretti F, Riggio V, Dall'Olio S, Calascibetta D, Russo V \& Portolano B 2010a Sequence characterization of the melanocortin 1 receptor $(M C 1 R)$ gene in sheep with different coat colour and identification of the putative e allele at the ovine Extension locus. Small Ruminant Research 91 200-207

Fontanesi L, Forestier L, Allain D, Scotti E, Beretti F, Deretz-Picoulet S, Pecchioli E, Vernesi C, Robinson TJ, Malaney JL, Russo V \& Oulmouden A 2010 b Characterization of the rabbit agouti signaling protein (ASIP) gene: Transcripts and phylogenetic analyses and identification of the causative mutation of the nonagouti black coat colour. Genomics 95 166-175

Fontanesi L, Dall'Olio S, Beretti F, Portolano B \& Russo V 2011 Coat colours in the Massese sheep breed are associated with mutations in the agouti signaling protein $(A S I P)$ and melanocortin 1 receptor $(M C 1 R)$ genes. Animal, doi:10.1017/S1751731110001382

Fontanesi L, Tazzoli M, Beretti F \& Russo V 2006 Mutations in the melanocortin 1 receptor $(M C 1 R)$ gene are associated with coat colours in the domestic rabbit (Oryctolagus cuniculus). Animal Genetics 37 489-493

Girardot M, Guibert S, Laforet M-P, Gallard Y, Larroque H \& Oulmouden A 2006 The insertion of a full-length Bos taurus LINE element is responsible for a transcriptional deregulation of the Normanne Agouti gene. Pigment Cell Research 19 346-355

Kijas JMH, Wales R, Törnsten A, Chardon P, Moller M \& Andersson L 1998 Melanocortin Receptor $1(M C 1 R)$ mutations and coat color in pigs. Genetics 150 1177-1785 
Klungland H, Våge DI, Gomez-Raya L, Adalsteinsson S \& Lien E 1995 The role of melanocyte-stimulating hormone $(\mathrm{MSH})$ receptor in bovine coat color determination. Mammalian Genome 6 636-639

Marklund L, Johansson Moller M, Sandberg K \& Andersson L 1996 A missense mutation in the gene for melanocyte-stimulating hormone receptor $(M C 1 R)$ is associated with the chestnut coat color in horses. Mammalian Genome 7 895-899

Maudet C \& Taberlet P 2001 Detection of cows' milk in goats's cheeses inferred from mitochondrial DNA polymorphism. Journal of Dairy Research 68 229-235

Maudet C \& Taberlet P 2002 Holstein's milk detection in cheeses inferred from melanocortin receptor 1 (MC1R) gene polymorphism. Journal of Dairy Science 85 707-715

Maudet C, Luikart G \& Taberlet P 2002 Genetic diversity and assignment tests among seven French cattle breeds based on microsatellite DNA analysis. Journal of Animal Science $\mathbf{8 0} 942-950$

Moio L, Sasso ML, Chianese L \& Addeo F 1990 Rapid detection of bovine milk in ovine, caprine and water buffalo milk or cheese by gel isoelectric focusing on PhastSystem. Italian Journal of Food Science $\mathbf{3}$ 185-191

Negrini R, Nicoloso L, Crepaldi P, Milanesi E, Colli L, Chegdani F, Pariset L, Dunner S, Leveziel H, Williams JL \& Ajmone Marsan P 2009 Assessing SNP markers for assigning individuals to cattle populations. Animal Genetics 40 18-26.
Norris BJ \& Whan VA 2008 A gene duplication affecting expression of the ovine ASIP gene is responsible for white and black sheep. Genome Research 18 1282-1293

Robbins LS, Nadeau JH, Johnson KR, Kelly MA, Roselli-Rehfuss L, Baack E, Mountjoy KG \& Cone RD 1993 Pigmentation phenotypes of variant Extension locus alleles result from point mutations that alter $\mathrm{MSH}$ receptor function. Cell 72 827-834

Royo LJ, Alvarez I, Arranz JJ, Fernández I, Rodríguez A, Pérez-Pardal L \& Goyache F 2008 Differences in the expression of the ASIP gene are involved in the recessive black coat colour pattern in sheep: evidence from the rare Xalda sheep breed. Animal Genetics 39 290-293

Russo V \& Fontanesi L 2004 Coat colour gene analysis and breed traceability. Proceedings of the 7th World Brown Swiss Conference, pp. 95-100, Verona, Italy.

Russo V, Fontanesi L, Scotti E, Tazzoli M, Dall'Olio S \& Davoli R 2007 Analysis of melanocortin 1 receptor (MC1R) gene polymorphisms in some cattle breeds: their usefulness and application for breed traceability and authentication of Parmigiano Reggiano cheese. Italian Journal of Animal Science 6 257-272

Searle AG 1968 Comparative Genetics of Coat Colour in Mammals. Logos Press, London, UK

Våge DI, Klungland H, Lu D \& Cone RD 1999 Molecular and pharmacological characterization of dominant black coat color in sheep. Mammalian Genome 10 39-43 